\title{
news
}

\section{news news news news news news news news}

\section{Guidelines to improve psychological and social support in emergencies}

A new set of guidelines to address the mental health and psychosocial needs of survivors of conflict and disaster have been agreed by international humanitarian agencies.

Under the 'Guidelines on mental health and psychosocial support in emergency settings' from the Inter-Agency Standing Committee (IASC), the protection and promotion of mental health and psychosocial well-being is positioned as the responsibility of all humanitarian agencies and workers, rather than only of psychiatrists and psychologists.

Armed conflicts and natural disasters can cause significant psychological and social suffering to affected populations, both in the short and long terms. One of the priorities in emergencies is to protect and improve people's mental health and psychosocial well-being. However, a significant gap has been the absence of a multi-sectoral, interagency framework that enables effective coordination and identifies useful practices, and these guidelines are aimed at filling this gap.

The guidelines cover the three phases of an emergency, i.e. emergency preparedness steps to be taken before emergen- cies occur, the minimum responses to be implemented during the acute phase of the emergency, and the comprehensive responses to be implemented once the minimum responses have been implemented, typically during the stabilised and early reconstruction phases of the emergency.

The guidelines, which are intended primarily for low- and middle-income countries, have a clear focus on social interventions and supports. They emphasise the importance of building on local resources such as teachers, health workers, healers, and women's groups to promote psychosocial well-being. They focus on strengthening social networks and building on existing ways community members deal with distress in their lives. They also include attention to the protection and care of people with severe mental disorders, including severe trauma-induced disorders, as well as access to psychological first aid for those in acute distress.

The guidelines stress that the way in which humanitarian aid is provided can have a substantial impact on people's mental health and psychosocial well-being. Treating survivors with dignity and enabling them to participate in and organise emergency support is essential.

The IASC is an inter-agency forum responsible for worldwide 
humanitarian policy and consists of heads of relevant UN and other intergovernmental agencies, Red Cross and Red Crescent agencies, and NGO consortia.

Source: www.who.int/mental_health/emergencies

\section{Nurses in mental health}

Africa, with only 0.32 nurses in mental health settings per 100000 population, has among the lowest proportion of mental health nurses globally, and well below the world average of 2.23 nurses per 100000 population. And these are divided almost equally between mental hospitals and psychiatric units in general hospitals, with none recorded in community mental health settings.

These are among the findings of the World Health Organization's first Atlas of Nurses in Mental Health 2007, which found that, in general, there are fewer mental health nurses per capita in low-income countries and that the level of training in low- and middle-income countries is lower than in high-income countries. Moreover, there are also fewer community mental health facilities in low- and middle-income countries. However, nurses have more authority to initiate and renew medication prescriptions in countries in Africa as well as in southeast Asia and the Western Pacific - in Africa more than $40 \%$ of nurses are allowed to prescribe psychotropic medicines - compared with $14 \%$ globally - and $57 \%$ are allowed to continue psychotropic medicine prescription.
Data gathered during the compilation of the Atlas suggest that the overall nursing shortage is a factor in explaining the insufficient numbers of nurses in mental health, with this shortage even more acute because of the lack of incentives for nurses to be trained to provide mental health services. There are also few financial incentives for nurses either to receive mental health training or to provide mental health services, while the stigma of mental illness also clearly contributes to the problem by limiting the number of nurses willing to make mental health nursing a career.

Based on the survey data and other available information, recommendations are:

- Recognise nurses as essential human resources for mental health care: Nurses, with appropriate training, can perform a much wider variety of functions within mental health services than they are currently allotted and their role ought to be expanded to incorporate assessment, clinical care and follow-up using psychosocial and pharmacological interventions. Nurses should be fully involved in the development of policy, plans and legislation and service programmes.

- Ensure that adequate numbers of trained nurses are available to provide mental health care: There is a need for more nurses with appropriate mental health training in low- and middle-income countries.

- Incorporate a mental health component into basic and post-basic nursing training: Mental health training is a necessary prerequisite for the provision of mental health care, but is also important for a holistic approach to general nursing care, and general nursing curricula need to be strengthened by incorporating appropriate mental health components.

Source: www. who.int 